\title{
Multi-channel Support for Dense Wireless Sensor Networking
}

\author{
Ozlem Durmaz Incel, Stefan Dulman, and Pierre Jansen \\ University of Twente, P.O. Box 217, 7500 AE Enschede, The Netherlands \\ \{durmazo, dulman, jansen\}@cs.utwente.nl
}

\begin{abstract}
Currently, most wireless sensor network applications assume the presence of single-channel Medium Access Control (MAC) protocols. When sensor nodes are densely deployed, single-channel MAC protocols may be inadequate due to the higher demand for the limited bandwidth. To overcome this drawback, we propose multiple channel support for improving the performance. Our method allows the nodes to utilize new frequency channels which results in the significant increase on the number of nodes that are granted access to the wireless medium. The method requires only one half-duplex transceiver per node, which is capable of sending and receiving over distinguished frequency channels. Simulation results show that, method successfully utilizes multiple channels and increases the performance proportional to the number of available frequencies for an example single-channel MAC protocol, LMAC.
\end{abstract}

\section{Introduction}

Wireless Sensor Networks (WSN) [1, is an evolving technology that is the fundament of various ubiquitous applications. WSN is embedded into the real world and enables monitoring, inspection and analysis of unknown, untested environments with battery operated, tiny sensor devices. Sensor nodes are designed to collect sensor data about the context and to transmit the readings by wireless communication.

With the growing interest, in the near future, WSN will be deployed everywhere in large numbers, which may be of the order of hundreds or thousands and even more [2]. The underlying protocols must be able to deal with these numbers of nodes. In a dense network, demand is higher for the limited bandwidth. This results in less chance to access the wireless medium due to higher contention in a dense neighborhood 1 . Besides the large numbers, limited channel capacity and the influence of interference due to external networks or electronic devices, that share the same parts of the spectrum, will result in a competitive communication environment.

\footnotetext{
${ }^{1}$ We define the neighborhood of a node as the set of nodes which are located within the node's transmission range. We consider a dense network where a node -on the average- has more than 50 nodes in its neighborhood.
} 
The important reason for this competition is that sensor nodes share a single channe 2 . If the transceiver equipment used for wireless communication is able to operate on multiple non-overlapping channels rather than a single channel, multiple transmissions can take place on the wireless medium without disturbing each other. This leads to lower contention, less collisions and retransmissions. Today's transceiver hardware, which is used for sensor nodes, supports operation on multiple frequencies. For example, the radio used by Ambient $\mu$ Node [3] and CC2420 radio 4] for MICAz and Telos sensor nodes can be tuned to different channels.

We consider the LMAC protocol [5, 6] as an example to show the inefficiency of single-channel MAC protocols in densely deployed sensor networks. LMAC is a light-weight and energy efficient MAC protocol proposed for WSN. It is based on the concept of scheduled access to the wireless medium. Each node controls a timeslot to transmit its data. Timeslots are selected in a distributed, selfconfiguring way. Further details of the LMAC protocol will be given in Section 3 .

Besides its advantages, LMAC's operation depends on the number of timeslots, and in turn on the density of the neighborhood. When all timeslots are exhausted, the node may not be able to access the wireless medium and remains in its initialization state to find an empty timeslot. As the neighborhood gets denser, the number of required timeslots grows rapidly. Therefore, we need a mechanism that reduces the contention in the neighborhood and allows a node to control a timeslot for transmitting its data.

We propose to multiplex the timeslots with the frequency domain for using the spectrum more efficiently. Note that this approach does not use different transceivers; instead one half-duplex transceiver is sufficient. The proposed method allows the nodes to switch their transceivers on new channels on-demand, if the network reaches a density limit. One may argue that switching to different channels by a half-duplex transceiver will cause disconnections in the network. However, our method optimizes connectivity, i.e., connects as many neighbors as possible via multiple channels.

The method is composed of two phases. In the first phase, nodes select timeslots according to the single-channel LMAC rules. In the second phase, nodes select timeslots and also channels to communicate on. The LMAC protocol ensures that a timeslot is only reused after at least 2-hops. Thus, the number of timeslots that a node can select is not only limited by the number of 1-hop neighbors but also by 2-hop neighbors. However, if multiple channels are available, nodes are allowed to select those timeslots that are occupied by their 2-hop neighbors on different channels. The second phase is based on this idea of utilizing different channels by allowing concurrent transmissions.

The rest of the paper is organized as follows: Section 2 summarizes related work. Section 3 introduces the LMAC protocol. Section 4 describes the multichannel support for LMAC protocol. Section 5] reveals the performance of the method by experimental simulations. Section [6] discusses some concluding remarks and suggestions for future work.

${ }^{2}$ A channel is defined to be a frequency range over which two nodes communicate. We will use the terms "channel" and "frequency" interchangeably in the text. 


\section{Related Work}

The channel assignment problem and multi-channel MAC protocols in wireless networks have been extensively studied. Usage of multiple channels in multi-hop ad hoc networks has been shown to increase the throughput considerably, by allowing concurrent transmissions on different non-overlapping channels [7], 8], [9], 10], 11], 12. Details about the algorithms and comparisons are explained by Mo et al,. 13 .

When we look into WSN domain, characteristics are quite different from the ad-hoc networks. A typical sensor device is usually equipped with a single halfduplex radio transceiver, which can not transmit and receive simultaneously, but can work on different channels separately. On the other hand, traditional wireless ad hoc networks usually assume more powerful radio hardware and multiple transceivers per node. For instance, typical bandwidth used by WSN is usually very limited (e.g., 50Kbps). Zhou et al. 14 showed why multi-channel MAC protocols which are based on IEEE 802.11 are not suitable for WSN with respect to the packet size, RTS/CTS mechanism and limited bandwidth. We show why single-channel MAC protocols are not efficient in densely deployed sensor networks, for an example single-channel MAC protocol.

Zhou et al., [14 recently introduced the MMSN multi-frequency MAC protocol especially designed for WSN. MMSN consists of two aspects: frequency assignment and medium access. In frequency assignment, each node is assigned a frequency for data reception. Hence, a node intending to transmit should know about the receiver's frequency. Broadcast packets are transmitted on a dedicated channel. Medium access is a combination of contention and scheduled operation. Our method is not a complete MAC protocol proposal, but it provides multiple channel support for an example single-channel MAC protocol: Timeslots are multiplexed with frequency domain on demand, if the number of timeslots in the neighborhood is exhausted on a single channel.

IEEE 802.15.4 standard [15] also provides multiple channels for Personal Area Networks (PAN). The idea is to use non-conflicting channels to identify different PAN's. This is different than our approach where we introduce multiple channels in a dense network when the demand for the limited bandwidth is higher.

\section{The LMAC Protocol}

LMAC [5] is an energy-efficient medium access protocol designed for WSN. The protocol enables the communicating entities to access the wireless medium on a time-scheduled basis over a single frequency channel. Time-scheduled method has a natural advantage of collision free medium access, which avoids wasting energy and time.

Like other time-scheduled MAC algorithms, LMAC also considers time to be divided into slots which are further organized into periodic frames. A node with the intention to transmit can take control of a timeslot. A node transmits a control message at the beginning of its timeslot to address the receiver nodes. 
Neighbor nodes must always listen at the beginning of a timeslot which contains information about the intended receivers, synchronization and the current timeslot. If neighbor nodes discover that they are not the intended receivers they turn off their power-consuming transceivers.

The timeslot selection mechanism in LMAC is fully distributed, thus needs no base-stations or central authorities to decide and allocate the timeslots to the nodes. In addition, the multi-hop nature of the WSN allows the timeslots to be reused.

For timeslot selection, the nodes use an algorithm based on local information only. Each node maintains a vector of length equal to the number of timeslots. This vector is used for storing the occupied slots within the 2-hop neighborhood. Initially, the vector is cleared. Nodes transmit information in the control message about those timeslots that the node considers to be occupied by itself and its 1 -hop neighbors. When a packet is received, the logical $O R$ operation is executed to update the information about the occupied slots in the neighborhood and the information is stored in the vector. If a node is not yet controlling any timeslot, it selects one from the free slots. This method ensures that a timeslot is only reused after at least 2-hops. The distributed algorithm for timeslot selection is shown in Figure 1. The node marked with "?" is searching for a timeslot and other nodes control the timeslots they are marked by. It receives the occupied slots information from the neighbors, executes the $O R$ operation and finds timeslot 7 as free and grabs it.

When there are no more free slots (i.e. in a dense neighborhood), the node remains in its initialization state, periodically monitoring frames to find an empty timeslot. Reserving a timeslot for each node or increasing the number of timeslots

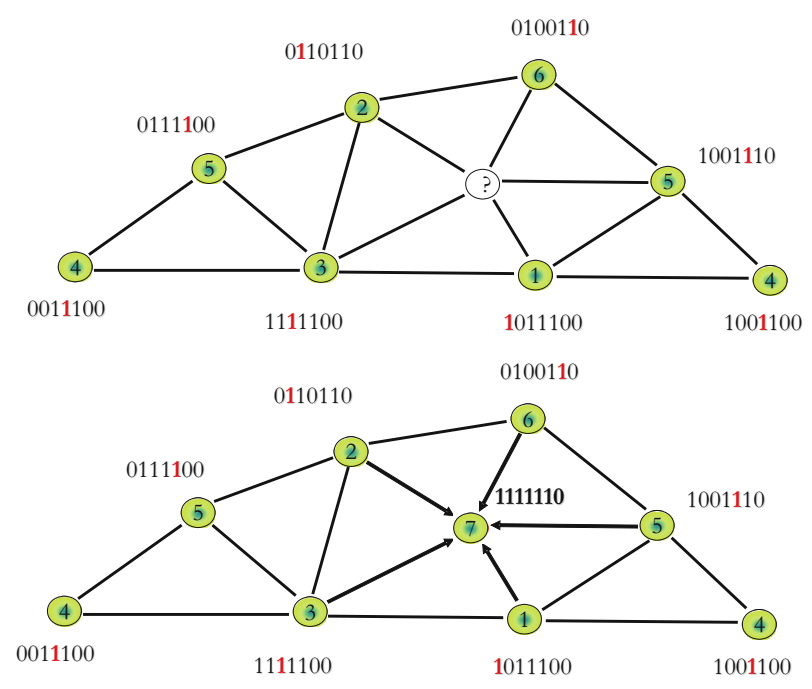

Fig. 1. LMAC Protocol: Timeslot selection 
in the network may be a possible solution. However, this would increase the latency of communication and time of waiting, before nodes get the opportunity to transmit. For this reason, the frame interval should be kept as short as possible and reused as much as possible. Currently, in a typical LMAC implementation, frame length is 1 second and a frame consists of 32 timeslots.

\section{Multi-channel Support for LMAC}

To overcome the deficiency of single-channel LMAC protocol in dense networks, we propose an algorithm which utilizes multiple channels. Besides, the algorithm optimizes connectivity, i.e., connects as many neighbors as possible.

The algorithms is based on local information only: it does not need central authorities to decide and allocate the frequencies. For instance, a central solution for multiple channel allocation -which is also used in cellular networks or DECTwould be to let a base-station (for example a sink node which is a gateway between the users of the network and the network itself in WSN) assign the channels. The sink node could assign frequencies to its 1-hop neighbors, and frequency information can be broadcast to the remaining nodes, in a multi-hop fashion. The nodes that are receiving the broadcast messages with frequency information, switch to the associated frequency. In this approach, the ultimate view of the network is partitions communicating on different frequencies. Nodes are only aware of their neighbors which are using the same channel. If two sensor nodes are within each other's neighborhood but they are not connected neighbors, the data packets that are destined for each other have to travel all the way from the sender to the sink node and from sink node to the destination node. This method would require a lot of messages to be relayed, and cause waste of energy and latency.

Before describing the algorithm in detail, we summarize the design issues and assumptions.

\subsection{Design Issues and Assumptions}

$-N$ non-overlapping frequency channels are available. Nodes are aware of the number and frequency range of the channels.

- All the nodes are communicating in the basic channel (single channel) at the beginning. If timeslots are exhausted in a node's neighborhood on the basic channel, new channels are introduced.

- The switching delay from one channel to another can be neglected, e.g., for the transceiver of Ambient $\mu$ Node sensor node platform, $650 \mu \mathrm{sec}$ is much less than a typical timeslot duration, $31.25 \mathrm{msec}$.

- Each node has one radio interface which is a half-duplex transceiver. A node cannot both transmit and receive at the same time, simultaneously.

- To establish multi-hop time synchronization ([16, [17]), every node uses its parent node to synchronize to every frame. A node can be a parent of another node if it's closer to the sink. 


\subsection{Functional Description}

The algorithm is composed of 2 phases. In the first phase, nodes select the timeslots according to the single-channel LMAC rules. In the second phase, channel selection takes place. The second phase is based on 2-hop neighborhood information. The LMAC protocol ensures that a timeslot is only reused after at least 2-hops. Thus, the number of timeslots that a node can select is not only limited by the number of 1-hop neighbors but also by 2-hop neighbors. Moreover, the simulation results (Section 5) have shown that on the average, more than $30 \%$ of the occupied slots of a node are claimed by its $2^{\text {nd }}$ hop neighbors. A node announces these 2-hop slots as free for its neighbors. However, the slots cannot be grabbed by a slotless node since all timeslots are occupied in its neighborhood. A slotless node cannot use the announced free slots on the same channel but can do so on a different channel. Therefore it monitors the announced free slots and marks the announcing node as a potential bridge. We call this node as a bridge node since it connects the slotless node to the rest of the network. A slotless node selects a bridge among the potential bridges and negotiates with the bridge on an appropriate slot/frequency pair. This negotiation keeps the network connected and the new joining node does not disturb the current established connectivity.

Figure 2 shows the idea of the second phase. In the figure, the slotless node is marked by a "?". Other nodes control the timeslots they are marked by. Node 3 announces its occupied vector as "1011" where node 4 is announcing as "0111". All timeslots in ?'s neighborhood are occupied $(1011$ OR $0111=1111)$. If a single-channel LMAC protocol was used, the node could not grab a slot in this situation. If multiple channels are available, after receiving this information, the slotless node views node 3 on slot 2 and node 4 on slot 1 as potential bridges. We explain the algorithm in the next sections based on the example given in Figure 2]

Bridge and non-interfering Channels Discovery. In the first phase of the algorithm, nodes are communicating on the basic channel and they are only aware of their neighborhood on that channel. However, before a slotless node started the second phase of searching for potential bridge nodes, other slotless nodes may have already connected to the network on different channels. In order to be aware of all potential bridges operating on different channels, a slotless node scans different channels for bridge node discovery. This process also helps the node to discover all the occupied timeslots in different frequencies before deciding on a non-interfering frequency/timeslot pair.

A slotless node creates two matrices about the collected information. One is for identifying the occupied slot/frequency pairs in the neighborhood. For the example shown in Figure 2, the slotless node creates the matrices shown in Figure 3. Here, number of timeslots is 4 and number of frequencies is 3 . In the occupied matrix, all the slots on the basic channel (frequency 0) are occupied where other channels are marked as free. In the free matrix, the slotless node records node 3 on slot 2 and and node 4 on slot 1 as potential bridges in any of the available frequencies other than the basic channel. After constructing the 
matrices, the slotless node extracts the required information about potential bridges. A bridge is selected due to some function from the set of potential bridges, such as the signal strength, degree of connectivity, battery level, etc.

Channel Negotiation. For the bridge node to be aware of a slotless node requesting negotiation, the slotless node should be able to send its request. However, it does not have a timeslot to transmit. One option for negotiation would be to take place on a dedicated control channel. Potential bridge nodes would switch to the control channel on slots which they announce to be free and listen for the requests. However, this would be very costly for the nodes in terms of energy if they have most of their timeslots as free. They would have to keep their transceiver always on, and probably receive no request. Instead, when a potential bridge node does not have data to send, it sends a notification for those slotless nodes which are interested in negotiation, during its control message (Section 3). In the rest of the timeslot, during data section the slotless node sends its request to the bridge node. This request includes the channel information on which the slotless node intends to communicate with the bridge. Here, a question may arise what if there are more slotless nodes which are intending to negotiate with this bridge node. To prevent collisions, nodes send the request based on a contention mechanism.

After getting the request, the bridge node also checks to investigate whether there are any conflicting transmissions on that slot/frequency pair in its neighborhood. It acknowledges the slotless node during its timeslot in the next frame if there is no conflict. After starting transmissions, still there may be collisions or interference with some other nodes. In this case, the agreement is canceled by the both parties and the requesting slotless node restarts the same process. For the example shown in Figure 2, let's assume that node? has agreed with

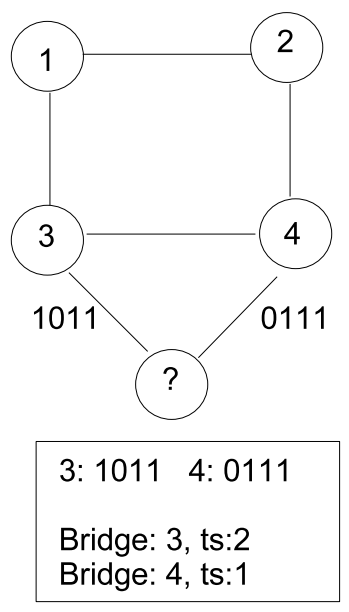

Fig. 2. Operation of the algorithm 
Occupied Matrix

\begin{tabular}{|c|c|c|c|}
\hline Timeslots/Frequencies & 0 & 1 & 2 \\
\hline 1 & occupied & - & - \\
\hline 2 & occupied & - & - \\
\hline 3 & Node 3 & - & - \\
\hline 4 & Node 4 & - & - \\
\hline
\end{tabular}

Free Matrix

\begin{tabular}{|c|c|c|c|}
\hline Timeslots/Frequencies & 0 & 1 & 2 \\
\hline 1 & occupied & Node 4 & Node 4 \\
\hline 2 & occupied & Node 3 & Node 3 \\
\hline 3 & occupied & - & - \\
\hline 4 & occupied & - & - \\
\hline
\end{tabular}

Fig. 3. Matrices discovered by node "?"

Node 1
\begin{tabular}{|c|c|c|c|c|}
\hline Frequency & $\mathbf{1}$ & $\mathbf{2}$ & $\mathbf{3}$ & $\mathbf{4}$ \\
\hline Neighbor & myslot & Node2 & Node3 & - \\
\hline Node 2 & $\mathbf{1}$ & $\mathbf{2}$ & $\mathbf{3}$ & $\mathbf{4}$ \\
\hline Frequency & 0 & 0 & - & 0 \\
\hline Neighbor & Node1 & myslot & - & Node4 \\
\hline Node 3 & $\mathbf{1}$ & $\mathbf{2}$ & $\mathbf{3}$ & $\mathbf{4}$ \\
\hline Frequency & 0 & 1 & 0 & 0 \\
\hline Neighbor & Node1 & Node ? & Node3 & - \\
\hline Node 4 & $\mathbf{1}$ & $\mathbf{2}$ & $\mathbf{3}$ & $\mathbf{4}$ \\
\hline Frequency & - & 0 & 0 & 0 \\
\hline Neighbor & - & Node2 & Node3 & myslot \\
\hline Node & $\mathbf{1}$ & $\mathbf{2}$ & $\mathbf{3}$ & $\mathbf{4}$ \\
\hline Frequency & - & 1 & 0 & - \\
\hline Neighbor & - & myslot & Node3 & - \\
\hline
\end{tabular}

Fig. 4. Occupied vectors with frequency information

node 3 on frequency 1 over slot 2 . The occupied vectors for the nodes are shown in Figure 4

After a slotless node has negotiated with a bridge node and is further controlling a timeslot, it can also play role as a potential bridge as well since it's already a part to the network. A sketch about execution model of multi channel LMAC is shown in Figure 5.

\section{Performance Evaluation}

In this section we present some experimental results concerning the inefficiency of LMAC protocol in dense networks, in terms of the number of active nodes (nodes that control a timeslot). Moreover, we investigate the overhead of using multiple channels. We have carried out simulations in the Omnet ++ environment [18]. 
The aim of doing experiments is to prove that the concept of the multi-channel support for LMAC protocol is valid.

Fixed simulation parameters are tabulated in Table 1. Sensor nodes are deployed randomly within the terrain, and are assumed to be static during the simulation interval. A topology generator tool is used to deploy sensor nodes randomly (with a uniform distribution) within the given dimensions of terrain size. We create 5000 random topologies and for each simulation run, (whether for LMAC or multi-channel LMAC version) the same topology is used. By changing the number of nodes but keeping the terrain size and the transmission range fixed, we simulate different levels of neighbor density. We adopt the neighbor density calculation from Bulusu et al. [19, as:

$$
\mu=\left(N \Pi R^{2}\right) / A
$$

where $\mathrm{N}$ is the number of nodes, $\mathrm{R}$ is the transmission range and $\mathrm{A}$ is the size of the terrain. So densities for different number of nodes $(50,100,150,200,250$, $300,350,400,450,500,550)$ are approximately $24,48,72,96,120,144,168$, 192, 216, 240 and 264. Note that these numbers include only 1-hop densities and don't include $2^{\text {nd }}$ hops. So, the neighbor densities in the example topologies are larger than these numbers and almost covering all of the nodes in the network.

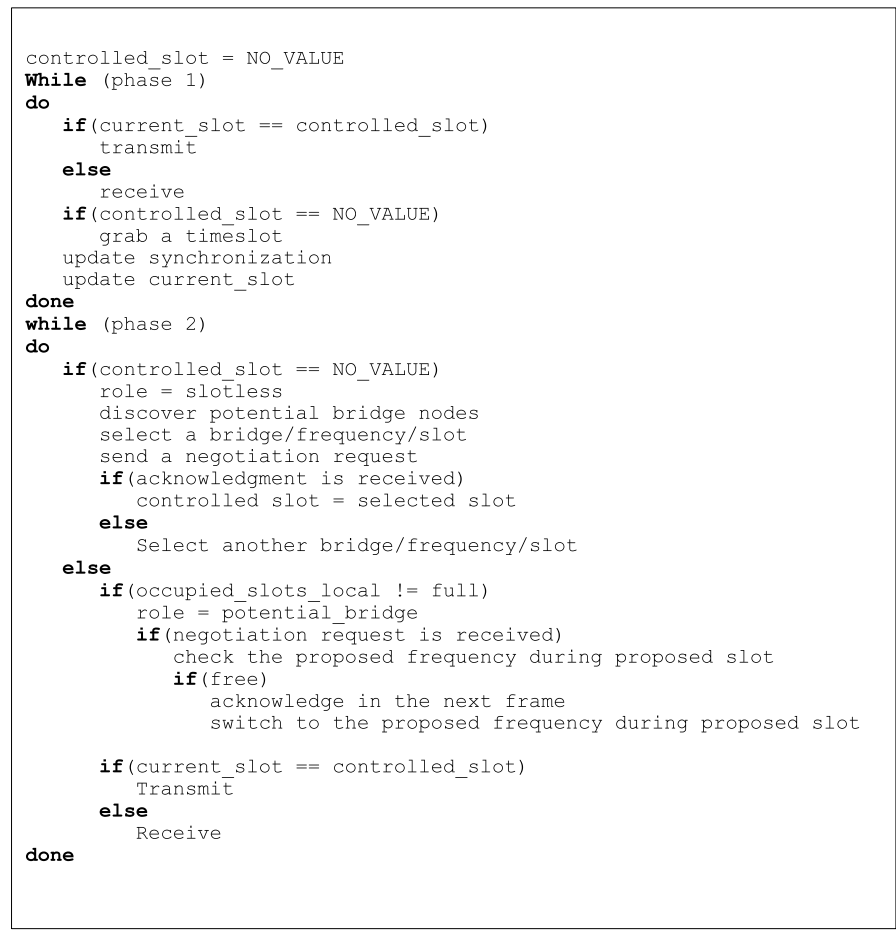

Fig. 5. Multi channel support for LMAC: execution model 
Table 1. Simulation Parameters

\begin{tabular}{||c|c||}
\hline \hline Parameter Name & Value \\
\hline Terrain Size & $100^{*} 100 \mathrm{~m}^{2}$ \\
Transmission Range & $40 \mathrm{~m}$ \\
Sensor Node Deployment & Uniform \\
Mobility Characteristic & Static \\
Density of Neighborhood & $24,48,72,96$ \\
& $120144,168,196$ nodes \\
Number of Time Slots & 32 timeslots \\
MAC frame size & 1 sec. \\
Number of Frequencies & 8 (non-overlapping) \\
Number of Runs & 5000 \\
\hline \hline
\end{tabular}

The traffic pattern used in the simulations is that every node broadcasts its occupied vector during its timeslot in its neighborhood. We assume error-free links. The simulation ends either when all the nodes control a timeslot or when there are no more timeslots that can be grabbed by a node. The simulation time limit is 100 seconds.

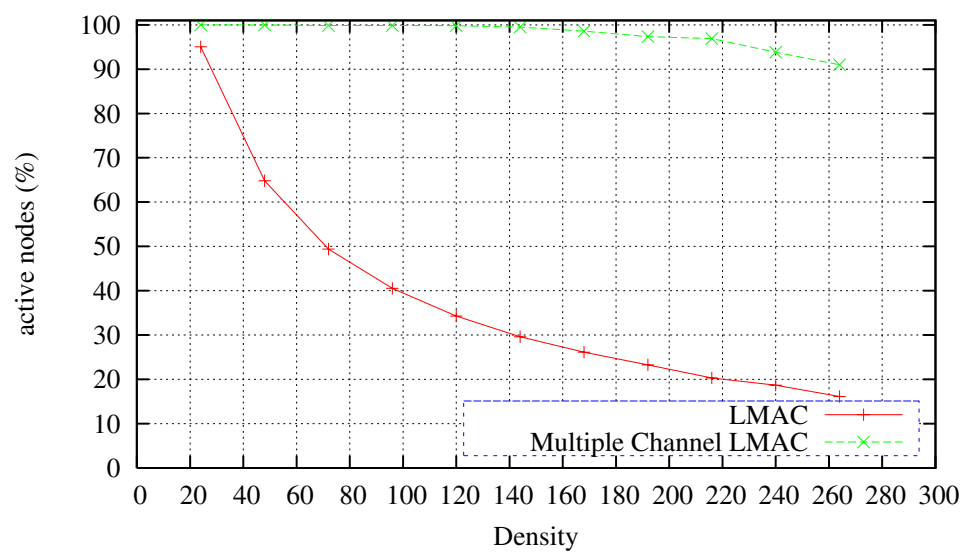

Fig. 6. Percentage of active nodes versus density

Firstly, we show how multiple channels increase the number of active nodes in the network. In Figure 6] the percentage of active nodes ([The nodes that have a timeslot/Number of nodes $\left.]^{*} 100\right)$ versus different number of densities is shown. When the network becomes denser, the percentage of active nodes decreases rapidly in LMAC. For 240 neighbors, it is below 20\%. Consequently, LMAC suffers from density. If we compare the results of multi-channel LMAC with pure LMAC's results we see that the performance is above $90 \%$ even in the most dense topologies. This method ensures that nodes can communicate, i.e. have a timeslot in a dense neighborhood even the neighborhood is getting denser with 
the increasing number of nodes and the same terrain dimensions. Multi-channel LMAC increases the performance by a factor of number of available channels. However, the number of active nodes is limited by "number of timeslots * number of frequencies". This shows that approximately all the channels are successfully utilized. In conclusion, LMAC's performance is affected by the number of timeslots whereas multi-channel LMAC increases the performance by the number of non-overlapping channels available.

To design a good MAC protocol for WSN, the first attribute to be considered is the energy efficiency [20] since the sensor nodes are usually battery-powered and it's difficult to change or recharge batteries. The major source of energy waste is the collisions. In this set of experiments we have tested how energyefficient our method is in terms of collisions. Another source of energy-waste is the control packet overhead. We test the overhead of the control packets sent during the negotiation process. The results are shown in Figures 7 and 8 . The collisions are represented per node for a fair comparison instead of totals. Because in LMAC number of active nodes is less and total number of collisions is also less. Number of collisions per active node in multi-channel LMAC is much less than LMAC. Multi-channel LMAC introduces multiple channels and this results in less number of collisions and contentions for timeslots. Control packets are not present in LMAC while in multi-channel LMAC, number of control packets increases with increasing density but it is still within boundaries between 0 and 5 per active node. These results are also represented in terms of control packets per node over a simulation period. The results prove that multi-channel LMAC does not bring an overhead in terms of energy.

Another parameter which directly affects the execution of the method is the number of available 2-hop slots offered by a node to be used by nodes in different frequencies. Results are shown in Figure 9, It's seen that in even most of the crowded topologies, more than $30 \%$ (10/32)of the slots are announced as free and slotless nodes can negotiate for different frequencies on these slots.

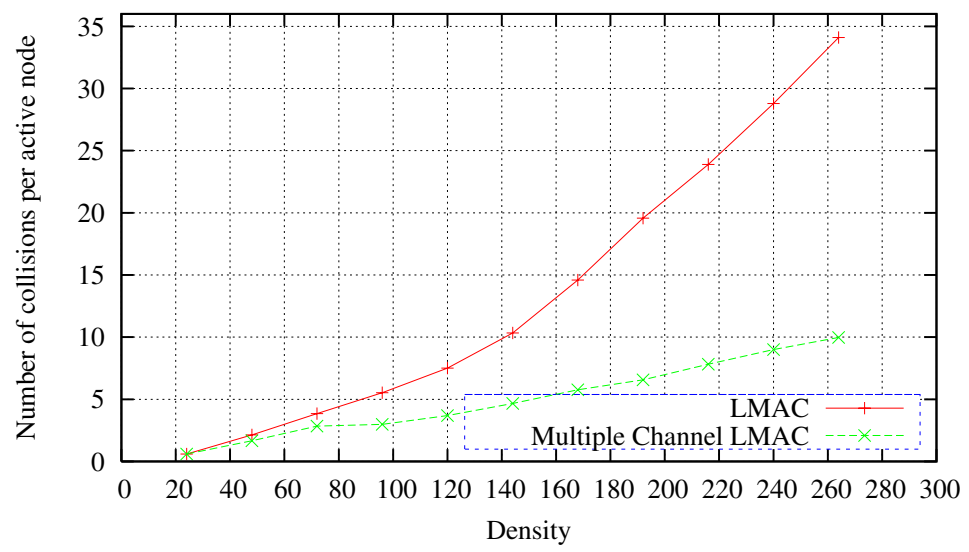

Fig. 7. Number of collisions per number of active nodes(\%) versus density 


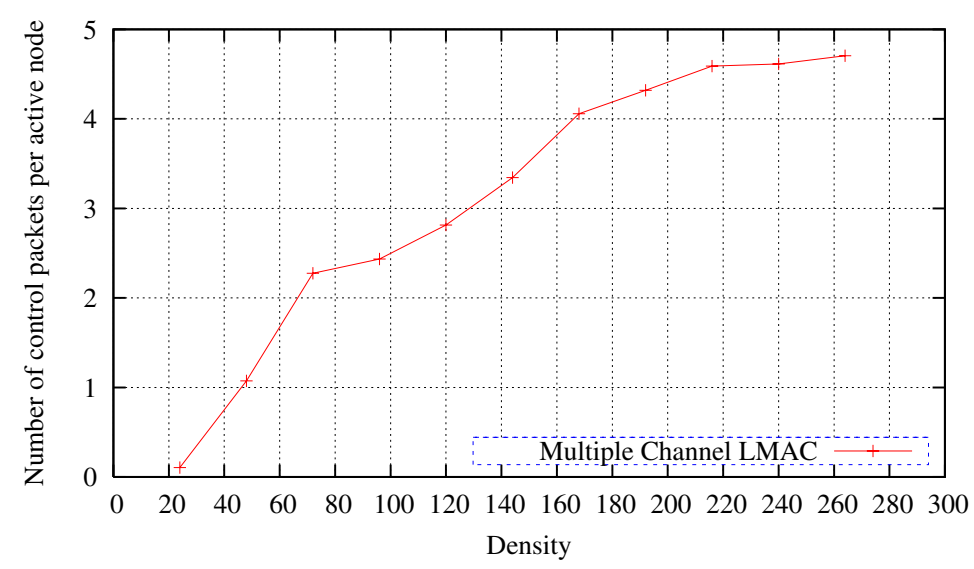

Fig. 8. Number of control packets per number of active nodes(\%) versus density

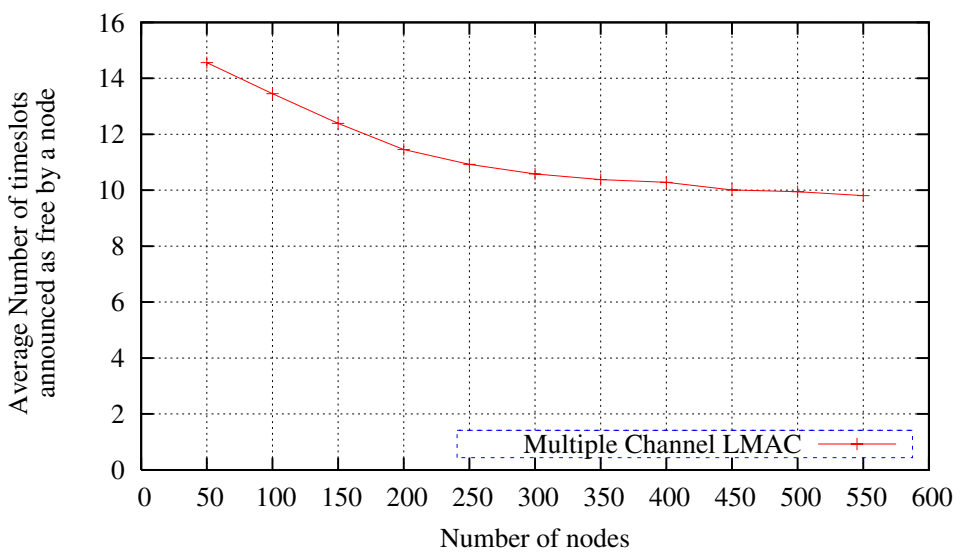

Fig. 9. Number of available slots announced by a node versus number of nodes

\section{Conclusion and Future Work}

In this future-looking work, we have addressed multiple channel support for WSN. The single-channel MAC protocols which grant access to the bandwidth may be inadequate in dense environments due to the higher demand and contention for the limited bandwidth. We propose a method that extends an example single-channel MAC protocol by multiplexing the time domain with the frequency domain. It allows the nodes to introduce new channels on-demand, if the network reaches a density limit. The method is composed of two phases. In the first phase, nodes try to select timeslots according to the single-channel LMAC rules. In the second phase, nodes that could not grab a timeslot in the 
first phase invite the neighbor nodes which are free to listen them on an agreed frequency channel and a timeslot.

The current state of the work proves that an example single-channel timeslotted MAC protocol "LMAC" suffers in dense environments and the performance can be increased by introducing the multiple frequency channels. Simulation results show that multiple channel support has the effect of increasing the performance by a factor of number available frequencies. Moreover, it does not bring an overhead in terms energy efficiency per node.

After the encouraging results, we will explore the adaptivity aspect of this solution and investigate the performance of multi-channels in other MAC protocols also with mobility scenarios. Currently, we are experimenting the method on a test-bed to have a clear idea about the number of non-overlapping channels available since the extent of spatial reuse is directly proportional to this number.

\section{Acknowledgments}

The authors gratefully acknowledge Sape Mullender for his help in revising the manuscript.

\section{References}

1. Akyildiz, I.F., Su, W., Sankarasubramaniam, Y., Cayirci, E.: A survey on sensor networks. IEEE Communications Magazine 40 (2002) 102-114

2. Gang Zhou, J.S., Son, S.: Crowded spectrum in wireless sensor networks. In: EmNets 2006: Proceedings of the Third Workshop on Embedded Networked Sensors. (2006)

3. http://www.ambient systems.net: Ambient-systems products line-up (2006)

4. http://www.chipcon.com: Cc2420, 2.4 ghz, ieee 802.15.4, zigbee-ready rf transceiver (2006)

5. van Hoesel, L., Nieberg, T., Wu, J., Havinga, P.: Prolonging the lifetime of wireless sensor networks by cross layer interaction. IEEE Wireless Communication Magazine 11 (2005) 78-86

6. Hoesel, L.v., Havinga, P.: A lightweight medium access protocol (LMAC) for wireless sensor networks. In: Proceedings of INSS04, Tokyo, Japan (2004)

7. Nasipuri, A., Zhuang, J., Das, S.: A multichannel csma-mac protocol for multihop wireless networks. In: Proceedings of IEEE Wireless Communications and Networking Conference (WCNC'99). (1999) 1402-1406

8. So, J., Vaidya, N.H.: Multi-channel mac for ad hoc networks: handling multichannel hidden terminals using a single transceiver. In: Proceedings of the 5th ACM international symposium on Mobile ad hoc networking and computing (MobiHoc '04). (2004) 222-233

9. Jain, N., Das, S., Nasipuri, A.: A multichannel csma mac protocol with receiverbased channel selection for multihop wireless networks. In: Proceedings of the IEEE IC3N. (2001) 432-439

10. Lin, C.Y.: A multi-channel mac protocol with power control for multi-hop mobile ad hoc networks. In: Proceedings of the 21st International Conference on Distributed Computing Systems (ICDCSW '01). (2001) 419 
11. Roy, S., Das, A., Vijayakumar, R., Alazemi, H., Ma, H., Alotaibi, E.: Capacity scaling with multiple radios and multiple channels in wireless mesh networks. In: Proceedings of the First IEEE Workshop on Wireless Mesh Networks(WiMesh). (2005)

12. So, H.S.W., Walrand, J.: Design of a multi-channel mac protocol for ad hoc wireless networks. Technical Report UCB/EECS-2006-17, EECS Department, University of California, Berkeley (2006)

13. Mo, J., Sheung, H., So, W., Walrand, J.: Comparison of multichannel mac protocols. In: Proceedings of the 8-th ACM/IEEE International Symposium on Modeling, Analysis and Simulation of Wireless and Mobile Systems. (2005) 209-218

14. Zhou, G., Huang, C., Yan, T., He, T., Stankovic, J.A., Abdelzaher, T.F.: Mmsn: Multi-frequency media access control for wireless sensor networks. In: Proceedings of IEEE Infocom. (2006)

15. http://www.ieee802.org/15/pub/TG4.html: Ieee 802.15 wpan task group (2006)

16. Elson, J., Estrin, D.: Time synchronization for wireless sensor networks. In: IPDPS '01: Proceedings of the 15th International Parallel \& Distributed Processing Symposium. (2001) 186

17. Elson, J., Roemer, K.: Wireless sensor networks: a new regime for time synchronization. SIGCOMM Comput. Commun. Rev. 33 (2003) 149-154

18. Varga, A.: Omnet++ community site (2006)

19. Estrin, D., Govindan, R., Heidemann, J., Kumar, S.: Next century challenges: scalable coordination in sensor networks. In: MobiCom '99: Proceedings of the 5th annual ACM/IEEE international conference on Mobile computing and networking. (1999) 263-270

20. Demirkol, I., Ersoy, C., Alagoz, F.: Mac protocols for wireless sensor networks: a survey. IEEE Communications Magazine 44 (2006) 115-121 